\title{
Circuit
}

Musiques contemporaines

\section{Trio Fibonacci : 20 ans et toutes ses cordes}

\section{Marc Hyland}

Volume 29, numéro 1, 2019

URI : https://id.erudit.org/iderudit/1059432ar

DOI : https://doi.org/10.7202/1059432ar

Aller au sommaire du numéro

\section{Éditeur(s)}

Circuit, musiques contemporaines

ISSN

1183-1693 (imprimé)

1488-9692 (numérique)

Découvrir la revue

Citer ce document

Hyland, M. (2019). Trio Fibonacci : 20 ans et toutes ses cordes. Circuit, 29(1),

81-82. https://doi.org/10.7202/1059432ar d'utilisation que vous pouvez consulter en ligne.

https://apropos.erudit.org/fr/usagers/politique-dutilisation/ 


\section{Trio Fibonacci : 20 ans et toutes}

\section{ses cordes}

Marc Hyland

Après vingt années de créations d'œuvres, de virtuosité ardente et de plus récentes fréquentations du grand répertoire consacré, les qualités musicales et d'engagement du Trio Fibonacci ne peuvent que faire consensus. J'aimerais ici ajouter ma voix à cette célébration de leurs noces de porcelaine.

La création d'un ensemble musical, en réunissant des interprètes et autant de personnalités, me semble convoquer le principe d'émergence, dont j'ai d'abord pris connaissance en entendant l'astrophysicien Hubert Reeves en faire mention lors d'une entrevue diffusée à la radio il y a plusieurs années. Le concept m’avait alors séduit, au point de refaire surface lors de la gestation de ce texte. Formalisé au xixe siècle, on pourrait le résumer par l'adage: «le tout est plus que la somme de ses parties». On peut qualifier une propriété d'«émergente» si elle tire son origine de propriétés plus fondamentales tout en demeurant «nouvelle» ou «irréductible» à celles-ci. Ainsi, les propriétés de l'eau seraient non réductibles à celles attribuées à l'hydrogène ou à l'oxygène. Les deux exemples les plus connus de phénomènes considérés comme émergents sont la conscience, comprise comme une propriété émergente du cerveau, et la vie, entendue comme une propriété émergente de la physique et de la chimie des organismes vivants. Je renvoie les lecteurs intéressés à

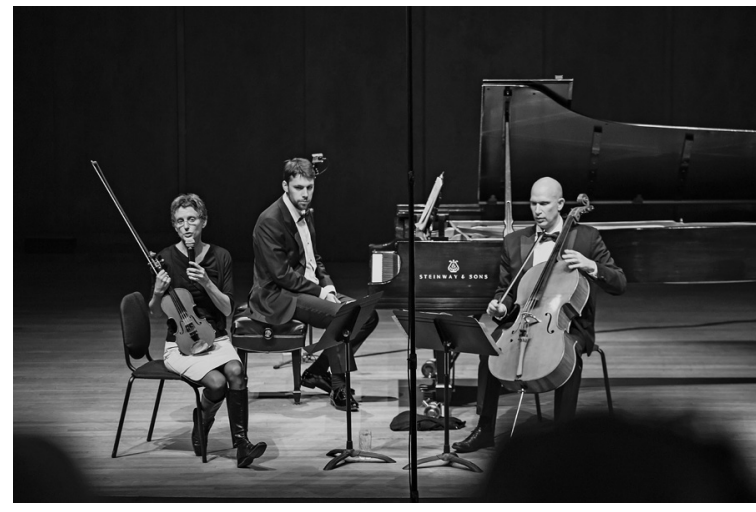

Le Trio Fibonacci (de gauche à droite: Julie-Anne Derome, Stephen Massicotte et Gabriel Prynn), à la salle Bourgie du Musée des beauxarts de Montréal, 2018. Crédit: Adrián Morillo.

approfondir cette notion aux écrits du philosophe John Stuart Mill, qui en a notamment jeté les bases dans son ouvrage A System of Logic (1862)1.

Le Trio Fibonacci me semble incarner musicalement ce principe, ce qui a pu contribuer à ce qu'il dure comme il le fait. Depuis vingt ans, le Trio compte deux éléments «stables», soit Julie-Anne Derome au violon et Gabriel Prynn au violoncelle, deux « cordistes » qui œuvrent depuis le début dans une complémentarité et un accord d'une constance et d'une profondeur exceptionnelles. Avec eux, un autre instrument à cordes - frappées celles-là -, le piano, dont les différents interprètes, au fil de l'existence de l'ensemble, incarnent un élément plus «instable», mais tout aussi fertile. La mouvance interne créée par cette succession de pianistes m’apparaît quasi providentielle! Le pianiste et compositeur André Ristic aura d'abord insufflé sa précision à la formation originelle pendant huit ans. Puis les sensibilités riches et contrastées des Jacynthe Riverin, Anna d'Errico et Hugues Leclère ont nourri 
l'ensemble pour de brefs intérims, avant que la présence de Wonny Song au sein du Trio, pendant sept ans, ne le mène vers sa composition actuelle et des répertoires plus hétérogènes. Comment ne pas trouver dans ce piano en constante mutation un formidable instrument de ressourcement pour le duo violon-violoncelle semblant voué, lui, à la permanence? Depuis un an, c'est le formidable Stephen Massicotte qui occupe la part du piano avec un raffinement et une plénitude qui sied particulièrement bien à l'ensemble, notamment dans ses interprétations d'œuvres de l'âge classique.

Par curiosité, j'évoquerai ici un ensemble célébrissime, le Trio Beaux-Arts, actif entre 1955 et 2008. C'est dans ce cas le fondateur et pianiste Menahem Pressler qui en a constitué le seul élément permanent, ou pour filer la métaphore, le «principe stable», plusieurs vio- lonistes et violoncellistes s'étant succédé à ses côtés au fil de ces cinquante-trois années d'existence.

Il arrive, pour le privilège des mélomanes et des compositeurs, que des noyaux de musiciens se forment et durent avec justesse, pertinence et panache. A contrario de l'éphémérité ambiante, le Trio Fibonacci représente une telle occurrence, lieu d'une propriété émergente remarquable. Depuis sa fondation en 1998, nous assistons au déploiement d'une pensée en croissance, à l'image de la série de Fibonacci, spirale sans fin émergeant ici de visées strictement modernistes, qui se diffractent depuis pour connaître d'autres siècles.

1. John Stuart Mill (1995[1862]), Système de logique déductive et inductive, Bruxelles, Mardaga. Voir également l'article de Timothy O'Connore et Hong Yu Wong (2012), "Emergent Properties", The Stanford Encyclopedia of Philosophy, https://plato.stanford.edu/ entries/properties-emergent/ (consulté le 17 janvier 2019).

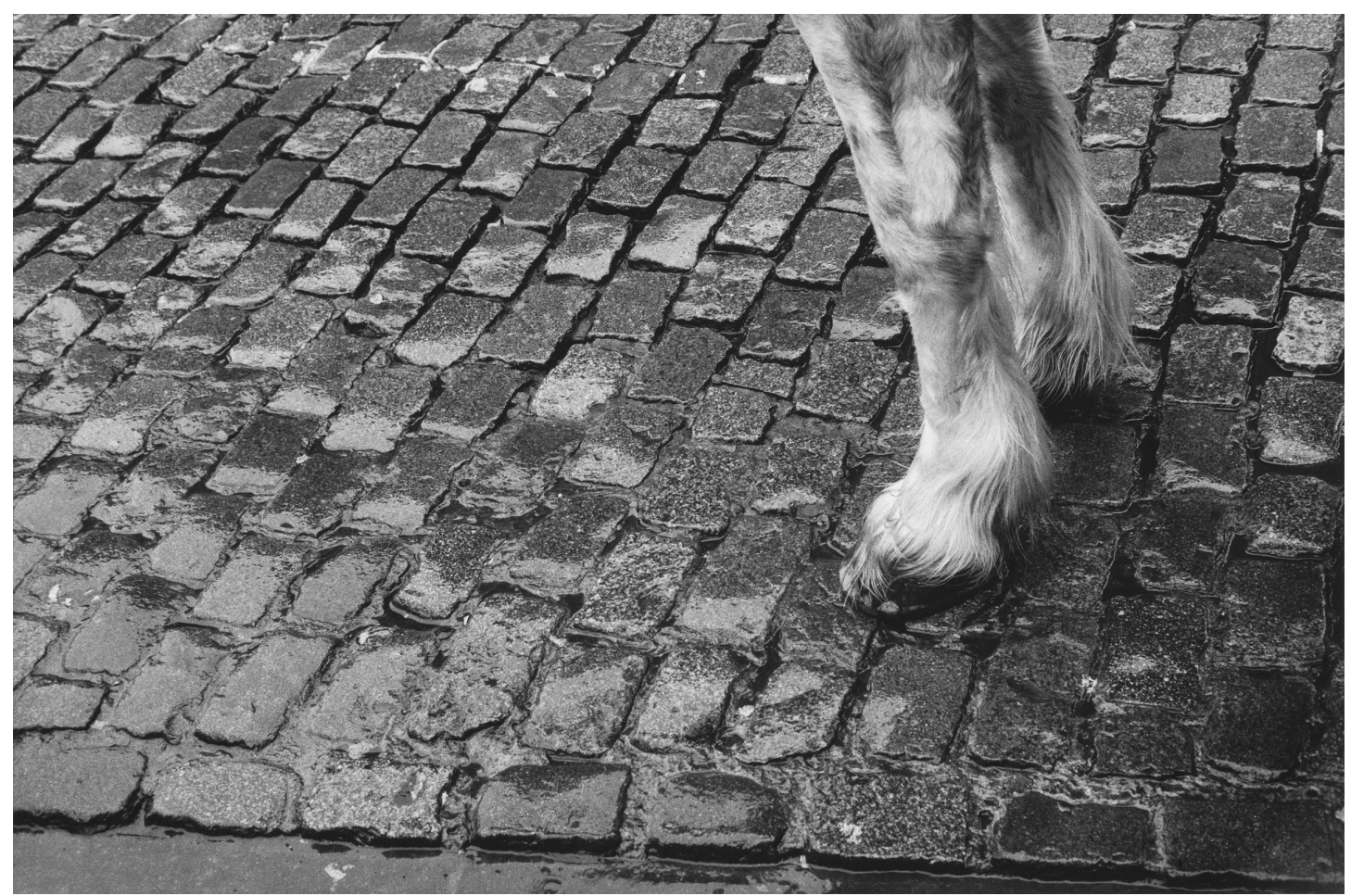

